\title{
Distinct Prognostic Factors in Sporadic and Multiple Endocrine Neoplasia Type 1-Related Pancreatic Neuroendocrine Tumors
}

Authors

Sapir Kon Kfir ${ }^{1}$, Reut Halperin 1, 2, Ruth Percik², 3, 4, Inbal Uri'2, 3, 4, Naama Halpern 4, 5, Gadi Shlomai1, 2, 4, Ido Laish4, 6 , Amir Tirosh2, 4, Amit Tirosh 2, 4, 7(1)

Affiliations

1 Department of Internal Medicine D, Sheba Medical Center at Tel Hashomer, Tel Hashomer, Israel

2 Division of Endocrinology and Metabolism, Sheba Medical Center at Tel Hashomer, Tel Hashomer, Israel

3 Endo-oncology Clinic, Cancer Center, Sheba Medical Center at Tel Hashomer, Tel Hashomer, Israel

4 Sackler Faculty of Medicine, Tel Aviv University, Tel Aviv, Israel

5 GI Unit, Cancer Center, Sheba Medical Center at Tel Hashomer, Tel Hashomer, Israel

6 Gastroenterology Institute, Sheba Medical Center at Tel Hashomer, Tel Hashomer, Israel

7 Neuroendocrine Tumors Service, Sheba Medical Center at Tel Hashomer, Tel Hashomer, Israel

Key words

MEN1, mortality, pancreas, neuroendocrine tumors

received 24.01.2021

accepted after revision $\quad 17.03 .2021$

published online 20.04.2021

Bibliography

Horm Metab Res 2021; 53: 319-325

DOI 10.1055/a-1464-1276

ISSN 0018-5043

(C) 2021. Thieme. All rights reserved.

Georg Thieme Verlag KG, Rüdigerstraße 14,

70469 Stuttgart, Germany

Correspondence

Amit Tirosh MD

Neuroendocrine Tumors Service, Chaim Sheba Medical

Center, Sackler Faculty of Medicine,

Tel Aviv University

Tel Aviv

Israel

Tel.: + 97235309236

amit.tirosh@sheba.health.gov.il
Supplementary material is available under

https://doi.org/10.1055/a-1464-1276

\section{ABSTRACT}

Pancreatic neuroendocrine tumors (PNET) may develop sporadically or in the context of hereditary syndromes. In patients with multiple endocrine neoplasia type 1 (MEN1), PNET is the leading cause of death. Our aim was to compare the mortality risk in sporadic and MEN1-related PNETs and identify high-risk populations. A retrospective Surveillance, Epidemiology, and End Results database analysis of patients with PNET was used. Patients with MEN1 were defined by syn/metachronous pituitary adenoma. Clinical data were retrieved, and all-cause mortality (ACM) risk was compared in univariate and multivariable analyses. The cohort included 569 patients ( $46.6 \%$ males) with sporadic $(n=542)$ and MEN1-related $(n=27)$ PNETs. Age at diagnosis of MEN1-related PNET was significantly younger than with sporadic PNETs (mean age 49.2 \pm 16.7 vs. $61.6 \pm 12.7$ years, respectively; $p<0.001$ ). Survival analysis showed a trend for a better outcome in patients with MEN1-related vs. sporadic PNET (Log-rank, $p=0.09$ ) and in subgroup analysis for patients with advanced disease $(p=0.08)$. Furthermore, among patients followed expectantly, those with MEN1-related PNET had lower ACM risk than their sporadic counterparts ( $p=0.08$ ). Multivariable analysis demonstrated lower ACM risk in patients diagnosed with MEN1 (hazard ratio 0.35 , 95\% confidence interval $0.11-1.2, p=0.09$ ), further supporting the trend detected in the univariate analysis. In conclusion, our study demonstrates the distinct clinical profile of patients with MEN1-related PNET compared to sporadic disease and emphasizes the expertise required to accurately manage patients with PNET in this rare context. The cautious decision-making required before embarking on surgical intervention is further emphasized in this robust analysis of a large cancer database.

\section{Introduction}

Multiple endocrine neoplasia type 1 (MEN1) is an autosomal dominant disorder caused by pathogenic germline variants in the MEN1 gene (11q13) encoding the menin protein [1]. The prevalence of MEN1 ranges between 1 and 10 in 100000 people in the general population [2]. The syndrome is characterized by the occurrence 
of primary hyperparathyroidism (PHPT) and pancreatic islet- and anterior pituitary tumors [3]. The most common manifestation of MEN1, occurring in almost all patients, is PHPT and is usually the earliest manifestation in patients, presenting before the 6th decade of life [4].

The reported prevalence for entero-pancreatic neuroendocrine tumors (NET) in MEN1 ranges between 30-80\% [5], while the rate of MEN1 among patients with pancreatic NET (PNET) varies depending on the functional status of the tumor, ranging between $3-15 \%$ in nonfunctioning PNET, to $20-25 \%$ in gastrin secreting PNETs [2]. The majority of MEN1-related PNET are gastrinomas, insulinomas, and nonfunctioning tumors [5]. Other less frequently detected PNET types in MEN1 include glucagonomas, vasoactive intestinal peptide secreting tumors, and somatostatinomas [5]. The reported prevalence of pituitary tumors among patients with MEN1 varies between 10 and $60 \%$, and it may be the first clinical manifestation in approximately $25 \%$ of de-novo cases [6]. Microscopically, MEN1-related PNETs occur in $80-100 \%$ of patients with MEN1. Hence they are often multiple, located throughout the pancreas but become symptomatic only in a minority of patients (0-13\%)[7]. Patients with MEN1 are prone to develop other neoplasms, including adrenal tumors, thyroid adenomas, thymic, gastric, and/or bronchial NET, brain meningiomas and ependymomas, skin lesions and smooth-muscle tumors, and also breast cancer $[8,9]$.

Diagnosis with MEN1 may be defined based on one of the following criteria: Clinical diagnosis, in the presence of two out of the three main MEN1-related manifestations (primary hyperparathyroidism, anterior pituitary tumor, and/or entero-pancreatic NET); Familial diagnosis, in the presence of one MEN1-related manifestation when at least one first-degree relative is also diagnosed with MEN1, or Genetic diagnosis, when a germline pathogenic variant is detected in the MEN1 gene [5].

Patients with MEN1 have shorter longevity compared with the general population [10]. In the past, Zollinger-Ellison syndrome (ZES) was the major cause of death in MEN1, but proton-pump inhibitors practically eliminated the catastrophic manifestations of ZES, such as perforated ulcers and massive upper gastrointestinal bleeding. This led to MEN1-related PNET becoming the main cause of death in this syndrome $[11,12]$. Yet, whether MEN1-related PNETs confer a higher mortality risk than their sporadic counterparts is still to be determined.

In the current work, we compared the mortality risk between sporadic and MEN1-related PNET and aimed to identify risk factors for mortality among patients with MEN1-related PNET to allow clinical identification of patients that may benefit from a more aggressive and early intervention.

\section{Materials and Methods}

This was a retrospective, population-based, case-control study based on the Surveillance, Epidemiology and End Results (SEER) database. The SEER database, maintained by the National Cancer Institute, provides cancer incidence and survival data from population-based registries, includes more than 3 million cases, and accounting for approximately $30 \%$ of the United States population. In the current study, we analyzed the November 2018 update providing information for cases diagnosed between 2000 and 2016 .
Data retrieved for the current analysis included patient demographics (age at diagnosis, gender, and ethnicity), tumor characteristics (stage, grade, and surgical intervention), and outcomes (survival since diagnosis and cause of death). The SEER database is publicly available, open for analysis by the scientific community, and patients' data are anonymized. Hence, institutional ethical approval was not required.

\section{Cohort construction}

The current analysis included patients documented in the SEER database, diagnosed with either pituitary adenomas or PNET of any type, either functional or non-functional. The diagnoses were sorted and identified based on the ICD-O-3 [13] histologic codes (Table 1S) and based on the NET anatomic site. Since diagnosis with pituitary adenoma and PNET defines MEN1, we identified patients with both diagnoses as those harboring MEN1-related PNET - the control group comprised of patients with PNET alone ('Sporadic PNET').

\section{Statistical analysis}

The analysis was performed on $\mathrm{R}$ Studio version 1.2.5001. Tumor grades were classified according to the SEER database definition, as the current definitions by the WHO for pancreatic neuroendocrine tumors grading have not been used during most of the study period and are not documented. Staging is reported based on the American Joint Cancer Classification (AJCC) 6th Edition [14] and according to the SEER staging definitions (localized, regional metastases, and distant metastases). Survival analysis was based on the SEER data and on the time since diagnosis until death or last follow-up. Continuous variables were compared using the Student's $t$-test, and categorical parameters were compared using the chisquare test. Continuous variables are presented as mean \pm standard deviation (SD) unless stated otherwise, and categorical parameters are presented as $\mathrm{n}(\%)$. Change in management efficacy over the study period was adjusted by defining the earliest year of diagnosis per group, and the latter year was set as a threshold for inclusion for both groups. Survival analysis was performed using the Kaplan-Meier method and compared by the log-rank test. Multivariable analysis was done using the proportional Cox regression analysis, controlling for covariates. A two-tailed $p$-value $<0.05$ was considered statistically significant.

\section{Results}

The study cohort comprised of 569 PNETs cases ( $56.6 \%$ male), 27 of them ( $4.7 \%$ ) have MEN1 based on clinical criteria. The mean age at diagnosis was $61.0 \pm 13.2$ years with a median of 62.0 [range $18.0,89.0]$. PNET were located at the head $(27.2 \%)$, body (13.5\%), or tail of the pancreas (31.5\%). Pancreatic-head NET were characterized by a higher grade ( $33.5 \%$ well-differentiated in the pancreatic head vs. $49.2 \%$ in body/tail PNET, $p<0.001$ ) and stage $(33.5 \%$ localized in pancreatic head NET vs. $50.8 \%$ in body/tail PNET, $p=0.001$ ), and surgical intervention were performed less often for pancreatic head versus body/tail PNET ( 45.8 vs. $67.6 \%, \mathrm{p}<0.001$, respectively). Considering the higher rate of PNET multiplicity on MEN1, we compared patients with a single record of PNET to those with multiple PNET records. When comparing multiple vs. single events, neither age at diagnosis, gender, stage, grade or surgical 
treatment were significantly different. However, a higher fraction of patients with multiple PNETs were of hispanic ancestry (28.6\%) compared to their representation among those with a single PNET $(8.9 \%, p=0.009)$. The cohort characteristics and comparison between patients with sporadic vs. MEN1-related PNET are detailed in Table 1.

\section{Univariate analysis - MEN1-related versus sporadic PNETs}

Age at diagnosis with MEN1-related PNET was significantly younger than with sporadic PNET (49.2 \pm 16.7 vs. $61.6 \pm 12.8$ years, respectively, $\mathrm{p}<0.001)$. PNET pancreatic location, grade, and stage were comparable between the MEN1 and sporadic groups ( $\triangleright$ Table 1 ).

\ Table 1 Demographic and tumor characteristics of entire cohort.

\begin{tabular}{|c|c|c|c|c|}
\hline & Overall & Sporadic & MEN1 & $\mathbf{p}$ \\
\hline $\mathrm{n}$ & 569 & 542 & 27 & \\
\hline MEN1 n (\%) & $27(4.7)$ & NA & NA & \\
\hline \multicolumn{5}{|l|}{ Age (years) } \\
\hline Mean (SD) & $61.00(13.24)$ & $61.59(12.78)$ & $49.22(16.72)$ & $<0.001$ \\
\hline Median (range) & $62.00(18.0,89.0)$ & $62.50(22.0,89.0)$ & $54.0(18.0,72.0)$ & $<0.001$ \\
\hline Male sex n (\%) & $322(56.6)$ & $305(56.3)$ & $17(63.0)$ & \\
\hline \multicolumn{5}{|l|}{ Ethnicity n (\%) } \\
\hline Hispanic & $55(9.7)$ & $51(9.4)$ & $4(14.8)$ & 0.627 \\
\hline Caucasian & $416(73.1)$ & $400(73.8)$ & $16(59.3)$ & 0.028 \\
\hline African American & $59(10.4)$ & $53(9.8)$ & $6(22.2)$ & 0.553 \\
\hline Asian or Pacific Islander & $35(6.2)$ & $35(6.5)$ & $0(0.0)$ & 0.15 \\
\hline Other & $4(0.7)$ & $3(0.6)$ & $1(3.7)$ & 0.081 \\
\hline Location in pancreas $\mathbf{n}(\%)$ & & & & 0.812 \\
\hline Head & $155(27.2)$ & $147(27.1)$ & $8(29.6)$ & \\
\hline Body & $77(13.5)$ & $75(13.8)$ & $2(7.4)$ & \\
\hline Tail & $179(31.5)$ & $170(31.4)$ & $9(33.3)$ & \\
\hline Other parts & $28(4.9)$ & $28(5.2)$ & $0(0.0)$ & \\
\hline Overlapping lesions & $42(7.4)$ & $39(7.2)$ & $3(11.1)$ & \\
\hline Unknown & $88(15.5)$ & $83(15.3)$ & $5(18.5)$ & \\
\hline Grade n (\%) & & & & 0.605 \\
\hline I (Well differentiated) & $231(40.6)$ & $217(40.0)$ & $14(51.9)$ & \\
\hline II (Moderately differentiated) & $66(11.6)$ & $62(11.4)$ & $4(14.8)$ & \\
\hline III (Poorly differentiated) & $23(4.0)$ & $22(4.1)$ & $1(3.7)$ & \\
\hline IV (Undifferentiated anaplastic) & $12(2.1)$ & $12(2.2)$ & $0(0.0)$ & \\
\hline Unknown & $237(41.7)$ & $229(42.3)$ & $8(29.6)$ & \\
\hline Stage $\mathbf{n}(\%)$ & & & & 0.257 \\
\hline Localized & $235(41.3)$ & $221(40.8)$ & $14(51.9)$ & \\
\hline Regional & $121(21.3)$ & $114(21.0)$ & $7(25.9)$ & \\
\hline Distant & $184(32.3)$ & $180(33.2)$ & $4(14.8)$ & \\
\hline Unknown & $29(5.1)$ & $27(5.0)$ & $2(7.4)$ & \\
\hline Stage AJCC n (\%) & & & & 0.558 \\
\hline 1 & $112(19.7)$ & 106 (19.6) & $6(22.2)$ & \\
\hline II & $260(45.7)$ & $246(45.4)$ & $14(51.9)$ & \\
\hline III & $70(12.3)$ & $66(12.2)$ & $4(14.8)$ & \\
\hline IV & $127(22.3)$ & $124(22.9)$ & $3(11.1)$ & \\
\hline Single event n (\%) & $548(96.3)$ & $522(96.3)$ & $26(96.3)$ & 1 \\
\hline Surgery performed n (\%) & $327(57.5)$ & $310(57.2)$ & $17(63.0)$ & 0.695 \\
\hline
\end{tabular}

MEN1: Multiple endocrine neoplasia type 1. NA: Not applicable. 
In terms of ethnic composition, a trend toward more patients of African American ancestry was found among patients with MEN1 (22.2\%) versus sporadic PNET (9.8\%, p =0.08). Nevertheless, when comparing patients of African American ancestry to other patients, other patient and tumor characteristics were comparable.

\section{All-cause mortality}

We did not find a significantly different risk for all-cause mortality (ACM) in our analysis. However, a trend for lower ACM was found for patients with MEN1-related vs. sporadic PNET (Log-rank test, $p=0.09$, Fig. 1) and especially among those with nonfunctioning PNET ( $p=0.07)$. A similar trend was found among patients with advanced disease, both based on the SEER staging system and based on AJCC staging, with lower ACM risk found in patients with MEN1-related versus sporadic PNET ( $p=0.08$ for both comparisons,

- Fig. 2). Comparison between patients with MEN1-related and sporadic PNET, demonstrated a trend for lower ACM in those that did not undergo surgical intervention ( $p=0.08$, > Fig. 3 ).

\section{Multivariable analysis}

We performed a multivariable Cox-regression analysis, adjusting for patient and tumor characteristics. In the adjusted survival analysis, age at diagnosis, tumor grade, and stage were all independently associated with increased risk for all-cause mortality ( $>$ Fig. 4). Again, a trend for lower ACM was found, even after adjusting for the covariates, in MEN1-related versus sporadic PNET (Hazard ratio $0.351,95 \%$ confidence interval $0.105-1.17, p=0.07$ ).

\section{Discussion}

In this study, we aimed to assess tumor and patient-related factors associated with increased risk for all-cause mortality in patients with sporadic and MEN1-related PNETs based on the SEER database. Our analysis demonstrated that patients with MEN1-related PNET are diagnoses at a younger age, as expected. The MEN1 group included a higher percentage of patients of African American ancestry compared with the sporadic PNET group. Although our com-
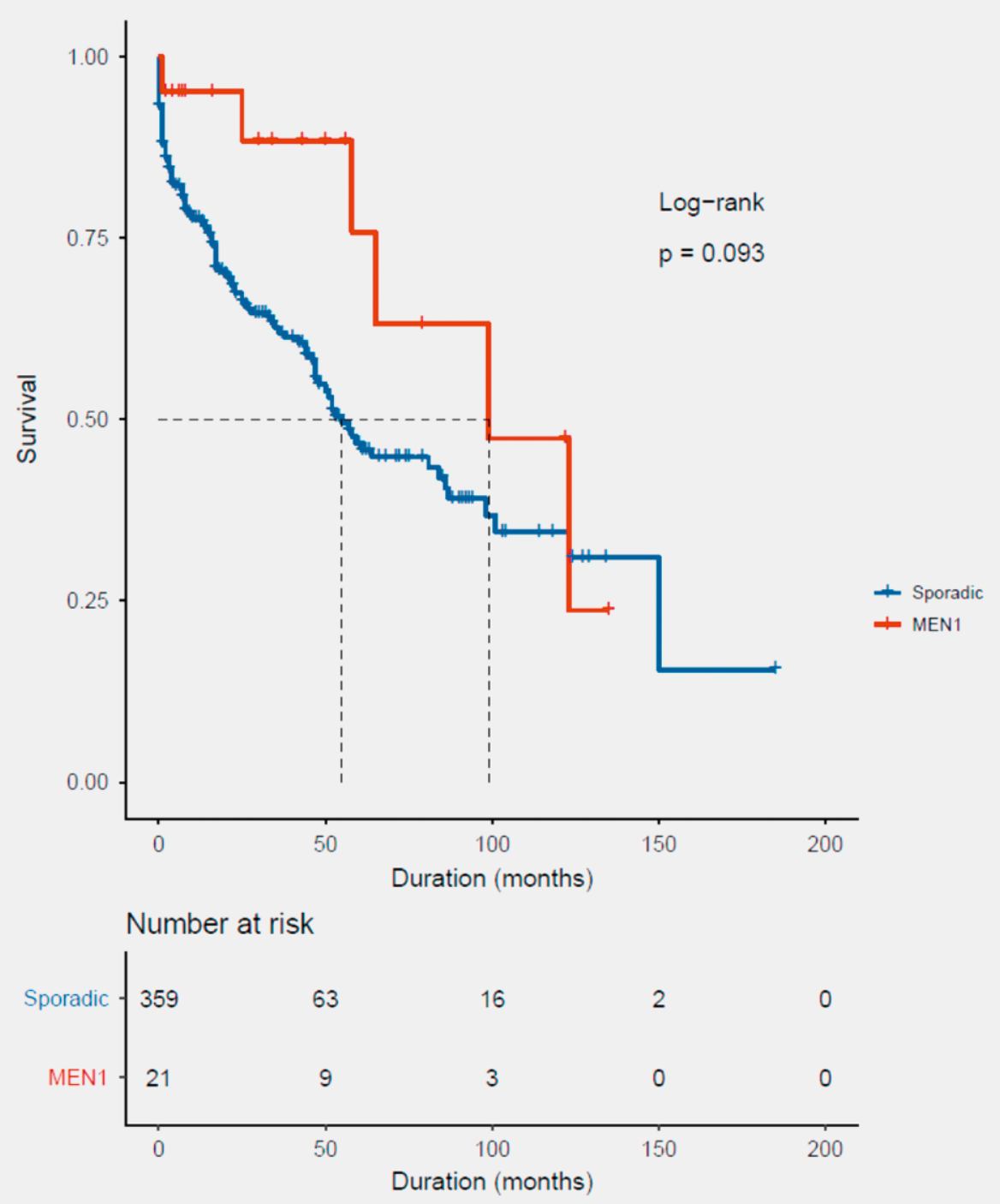

- Fig. 1 Kaplan-Meier analysis, comparing all-cause mortality risk between patients with MEN1-related and sporadic pancreatic neuroendocrine tumors. MEN1: Multiple endocrine neoplasia type 1. 


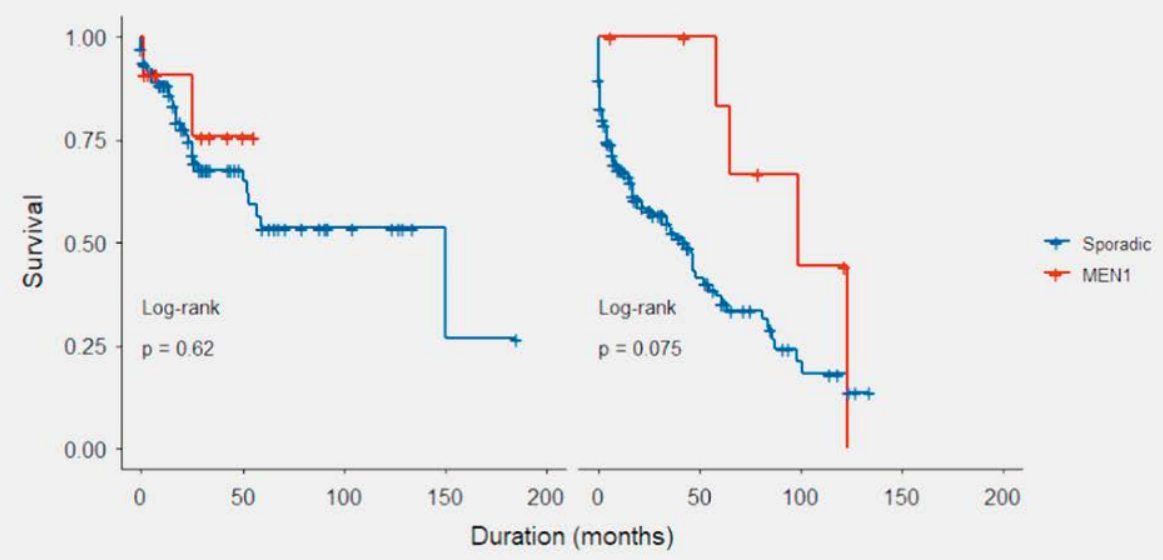

- Fig. 2 Kaplan-Meier analysis, comparing all-cause mortality risk between patients with MEN1-related and sporadic pancreatic neuroendocrine tumors - stratified by disease stage (localized disease - left, advanced disease - right). MEN1: Multiple endocrine neoplasia type 1.

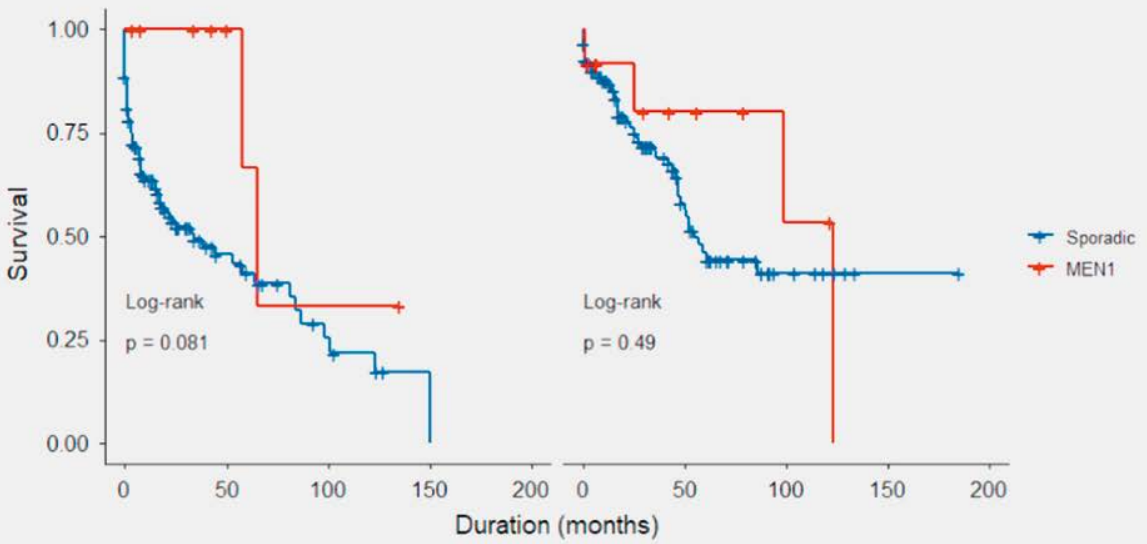

- Fig. 3 Kaplan-Meier analysis, comparing all-cause mortality risk between patients with MEN1-related and sporadic pancreatic neuroendocrine tumors - stratified to patients in which surgical intervention was performed (right) or not (left). MEN1: Multiple endocrine neoplasia type 1.

parative survival analysis did not reach statistical significance, the MEN1 group had a consistent trend for lower all-cause mortality risk across most analyses, including patients with advanced disease (AJCC stage III/IV). Importantly, among patients that did not undergo surgical resection of their tumor, those with MEN1-related PNET had better outcome compared to those with sporadic PNET. The trend for lower mortality risk in MEN1-related PNET was also found in the multivariable analysis.

Our finding that patients with MEN1-related PNET were diagnosed at a younger age compared to those with sporadic PNET is expected as has been previously reported $[7,15,16]$ and provides support for our cohort validity. Similarly, the increased risk for ACM by higher grade and stage in the multivariable analysis supports our study results.

We found that among patients that were followed expectantly (with no surgical intervention), those with MEN1-related PNET had lower ACM rates than sporadic PNET. A possible explanation is the low risk of small MEN1-related PNET to advance over time, as shown in previous studies of the Dutch and French MEN1 cohorts [17]. Our results support the conservative, non-invasive approach in patients with MEN1-related NET in the lack of clear benefit. Previously, the French research group of MEN1 showed that MEN1 patients with small NF-PNETS who undergone pancreatic surgery did not differ significantly in tumor progression compared to conservative treatment, and therefore suggested that surgery is not beneficial in this population [18].

Our study demonstrates once more that NETs in the context of hereditary syndromes are distinct from their sporadic counterparts. The genetic predisposition leads to clinical differences that should be translated into syndrome-specific clinical management. Specifically, we demonstrated that in patients with MEN1-related PNET, the risk for all-cause mortality may be lower compared to patients with sporadic PNET. Our analyses did not reach statistical significance, explained by the small sample size, but were consistent over several subgroup analyses and in the multivariable model. 


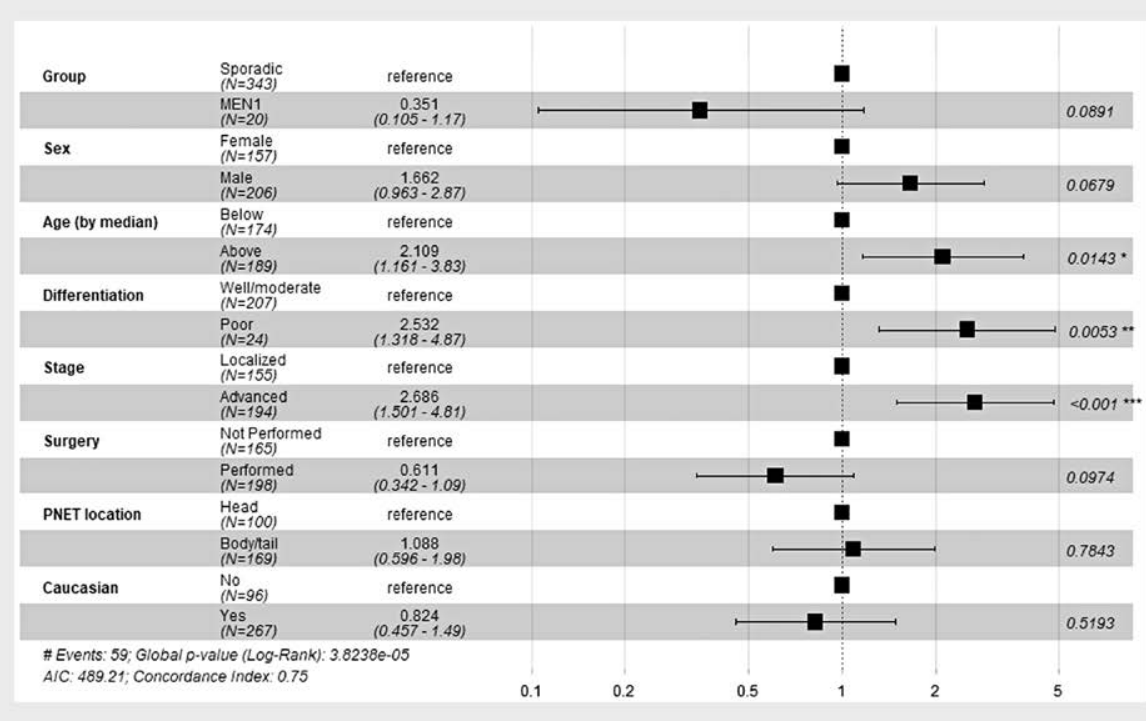

Fig. 4 Multivariable analysis for all-cause mortality in patients with sporadic and MEN1-related pancreatic neuroendocrine tumors. MEN1: Multiple endocrine neoplasia type 1 .

Hence, we advise choosing a cautious approach when considering surgical intervention in patients with MEN1-related PNET.

This study is based on one of the largest cancer databases available. The SEER database is a population-based cohort, representing the US population in an unbiased way. The data available enabled us to perform a robust multivariable analysis and to define the differences in the risk for mortality between MEN1-related and sporadic PNET. Our study had several unavoidable limitations that should be considered when interpreting our results. First, this study was a retrospective study, with all the inherent limitations and biases of this methodology. Second, our study may inaccurately represent the general MEN1 population for three reasons: 1 . Small sample size, 2. Possible inclusion of MEN1 phenocopies manifesting with PA and PNET, such as MEN4, and 3. Defining MEN1 patients based on PNET and PA co-occurrence and not based on parathyroid adenomas or family history, which are not reported in the SEER database. Third, since MEN1 genotype is not reported in the SEER database, the possible association between MEN1 mutation status and life expectancy could not be assessed [19]. Finally, we could not adjust for other comorbidities that may affect mortality risk.

In conclusion, based on a large cancer database, and while considering the above mentioned limitations we provide further validation to the less aggressive behavior of MEN1-related PNET, that while did not reach statistical significance, support previous studies, and may reflect on our clinical approach with patients suffering from this unique and rare syndrome.

\section{Conflict of Interest}

The authors declare that they have no conflict of interest.
References

[1] Norton JA, Krampitz G, Jensen RT. Multiple Endocrine Neoplasia: Genetics and Clinical Management. Surg Oncol Clin N Am 2015; 24: 795-832

[2] Jensen RT, Norton JA. Treatment of Pancreatic Neuroendocrine Tumors in Multiple Endocrine Neoplasia-Type 1(MEN1): Some Clarity but Continued Controversy. Pancreas 2017; 123: 4757-4763

[3] Thakker RV, Newey PJ, Walls GV et al. Clinical Practice Guidelines for Multiple Endocrine Neoplasia Type 1 (MEN1). J Clin Endocrinol Metab 2012; 97: 2990-3011

[4] Eller-Vainicher C, Chiodini I, Battista C et al. Sporadic and MEN1-related primary hyperparathyroidism: Differences in clinical expression and severity. J Bone Miner Res 2009; 24: 1404-1410

[5] Brandi ML, Gagel RF, Angeli A et al. CONSENSUS: Guidelines for Diagnosis and Therapy of MEN Type 1 and Type 2. J Clin Endocrinol Metab 2001; 86: 5658-5671

[6] Carty SE, Helm AK, Amico JA et al. The variable penetrance and spectrum of manifestations of multiple endocrine neoplasia type 1. Surgery 1998; 124: 1106-1114

[7] Jensen RT, Berna M], Bingham DB et al. Inherited pancreatic endocrine tumor syndromes: advances in molecular pathogenesis, diagnosis, management and controversies. Cancer 2008; 113: 1807-1843

[8] Brandi ML, Bordi C, Tonelli F et al. Multiple Endocrine Neoplasia Type 1. Princ Bone Biol Two-Volume Set 2008; 2: 1345-1374

[9] Van Leeuwaarde RS, Dreijerink KM, Ausems MG et al. MEN1-dependent breast cancer: Indication for early screening? Results from the Dutch MEN1 study group. J Clin Endocrinol Metab 2017; 102: 2083-2090

[10] Ito T, Igarashi H, Uehara $\mathrm{H}$ et al. Causes of Death and Prognostic Factors in Multiple Endocrine Neoplasia Type 1. Medicine (Baltimore) 2013; 92: 135-181

[11] Dean PG, Van Heerden JA, Farley DR et al. Are patients with multiple endocrine neoplasia type I prone to premature death? World J Surg 2000; $24: 1437-1441$ 
[12] Geerdink EAM, Van der Luijt RB, Lips CJM. Do patients with multiple endocrine neoplasia syndrome type 1 benefit from periodical screening? Eur J Endocrinol 2003; 149: 577-582

[13] World Health Organization. International Classification of Diseases for Oncology. Third Edition First Revision 2013

[14] Amin MB, Edge SB. American Joint Committee on Cancer. AJCC Cancer Staging Manual. In Internet: http://www.springer.com/us/ book/9783319406176; Stand: 06.11.2017

[15] Yates CJ, Newey PJ, Thakker RV. Challenges and controversies in management of pancreatic neuroendocrine tumours in patients with MEN1. Lancet Diabetes Endocrinol 2015; 3: 895-905

[16] Chiloiro S, Lanza F, Bianchi A et al. Pancreatic neuroendocrine tumors in MEN1 disease: A mono-centric longitudinal and prognostic study. Endocrine 2018; 60: 362-367
[17] Artic L, Pieterman CRC, De Laat JM De et al. Long-Term Natural Course of Small Nonfunctional Pancreatic Neuroendocrine Tumors in MEN1. Results From the Dutch MEN1 Study Group 2017; 102: 3795-3805

[18] Triponez F, Goudet P, Dosseh D et al. Is surgery beneficial for MEN1 patients with small $(\leq 2 \mathrm{~cm})$, nonfunctioning pancreaticoduodenal endocrine tumor? An analysis of 65 patients from the GTE. World J Surg 2006; 30: 654-662

[19] de Laat JM, van der Luijt RB, Pieterman CRC et al. MEN1 redefined, a clinical comparison of mutation-positive and mutation-negative patients. BMC Med 2016; 14: 1-9 